PROCEEDINGS OF THE

AMERICAN MATHEMATICAL SOCIETY

Volume 30, No. 1, September 1971

\title{
PRODUCTS OF NORMAL SUPERSOLVABLE SUBGROUPS
}

\author{
D. K. FRIESEN ${ }^{1}$
}

ABSTRACT. It is shown in this paper that if $G$ is a finite group which is the product of two normal supersolvable subgroups of relatively prime index, then $G$ is supersolvable.

In 1953, Huppert [1] gave an example showing that, in general, the product of two normal supersolvable subgroups of a finite group need not be supersolvable. The purpose of this note is to show that if the subgroups have relatively prime index in their product, then the product is again supersolvable. ${ }^{2}$

TheOREM. Let $A, B$ be normal supersolvable subgroups of the finite group $G$ with $G=A B$. If the indices $[G: A]$ and $[G: B]$ are relatively prime, then $G$ is supersolvable.

Proof. Assume that the theorem is false, and that $G$ is a counterexample of minimal order. Thus we may assume that the indices of $A$ and $B$ in $G$ are distinct primes $p$ and $q$ respectively with $A / A \cap B$ cyclic of order $q$ and $B / A \cap B$ cyclic of order $p$. Let $\alpha(A \cap B)$ and $\beta(A \cap B)$ generate these quotients and assume that $\alpha$ has order $q^{s}$ and $\beta$ has order $p^{t}$ for some $s, t \geqq 1$.

Let $E_{1}$ be a minimal normal subgroup of $A$ contained in $A \cap B$. If $A \cap B$ is trivial, the result follows immediately so $E_{1}$ is nontrivial and has order a prime $r$. If $E_{1}$ is normal in $G$, then by the minimality of $G$, we must have $G / E_{1}$ supersolvable; and since $E_{1}$ is cyclic, $G$ itself must be supersolvable. Thus $E_{1}$ has $p$ distinct conjugates $E_{1}$, $E_{2}, \cdots, E_{p}$. Then each $E_{i} \triangleleft A$ and hence $E=\left\langle E_{1}, E_{2}, \cdots, E_{p}\right\rangle$, the group generated by the $E_{i}$ is an elementary abelian $r$-group of order $\leqq r^{p}$, and $E \triangleleft G$. Among the subgroups of $E$ that are normal in $G$, select one, $L$, of minimal order. Since $A$ is supersolvable, $L$ has a cyclic normal subgroup, $U$, of order $r$, that is, $U$ is a one-dimensional $A Z_{\mathrm{r}}$-module. Then either $L=U$ or $L=U \oplus \alpha U \oplus \cdots \oplus \alpha^{p-1} U$. For $\alpha^{i} U \leqq U \oplus \cdots \oplus \alpha^{i-1} U$ implies that $U \oplus \cdots \oplus \alpha^{i-1} U$ is a $G$ invariant submodule of $L$ contradicting minimality of $L$ unless

Received by the editors September 30, 1970.

AMS 1970 subject classifications. Primary 20D40.

Key words and phrases. Supersolvable group, formation.

1 Research supported by NSF grant GP-7506.

2 The author wishes to express his appreciation to Professors W. R. Scott, J. T. Buckley, and M. Billis for helpful discussions.

Copyright @ 1971, American Mathematical Society 
$U \oplus \cdots \oplus \alpha^{i-1} U=L$. But then if $p$ does not divide $i$, there exist integers $m$ and $n$ such that $\alpha^{m p+n i}=\alpha$ and thus $\alpha U=\alpha^{n i} \alpha^{m p} U=\alpha^{n i} U$ $=U$. Since $i \leqq p$, we must have $i=p$ and the order of $L$ is $r^{p}$ or $r$. By similar reasoning applied to the subgroup $B$, the order of $L$ must be $r^{q}$ or $r$. Hence it must be $r$ and $L$ is cyclic. Since $G / L$ is supersolvable by induction, $G$ itself must be supersolvable and the theorem is proved.

REMARK 1. A consequence of the proof of the theorem is that in cases where the product $A B$ is not supersolvable for normal supersolvable $A$ and $B$, not only are $[G: A]$ and $[G: B]$ not relatively prime, but there is some prime $p$ such that $p|[G: A], p|[G: B]$, and $p|| A \cap B \mid$. Thus $p^{3}|| G \mid$. Indeed it is not difficult to show that any product of normal supersolvable subgroups which is not supersolvable contains a split extension of an elementary abelian $r$-group $R$ by a nonabelian $p$-group $P$ such that $P$ acts irreducibly on $R$.

REMARK 2. The fact that the conclusion of the theorem is also valid if supersolvable is replaced by nilpotent or cyclic suggests that the proper setting for the result is the theory of formations. However, the proof given above strongly uses the fact that a supersolvable group has a cyclic normal subgroup. Moreover, the following examples show that the result does not hold for arbitrary formations nor for saturated formations.

EXAMPLE 1. Let $F_{1}$ be the formation generated by $E_{1}$, the nonabelian group of order 14 , and $E_{2}$, the nonabelian group of order 21 . Then $E$, the holomorph of the cyclic group of order 7 , is not in $F_{1}$ although its subgroups of index 2 and 3 are normal and in $F_{1}$.

EXAMPLE 2. Let $r$ be any fixed prime $\neq 2,3,7$, and let the group $E$ of Example 1 have a faithful representation of degree $n$ over $\mathrm{GF}(r)$. Let this representation determine the action of $G$ in the semidirect product of $R$, the elementary abelian $r$-group of order $r^{n}$, and $E$ and let $G_{1}$ and $G_{2}$ be the subgroups of $G$ of index 2 and 3 respectively. Let $F$ be the saturated formation defined locally at each prime $p$ as follows:

For $p=2,3$ let $F(p)$ be the formation consisting of the group of order 1 .

For $p=7$, let $F(p)$ be the formation generated by the cyclic group of order 6 .

For $p=r$, let $F(p)$ be the formation $F_{1}$ of Example 1 .

For $p \neq 2,3,7, r$, let $F(p)$ be the empty formation.

Then $F$ is a saturated formation, $G_{1}, G_{2} \in F$ but $G \notin F$.

Presumably one can readily adapt the above proof to some formations contained in the formation of supersolvable groups. How- 
ever, since the result seems to shed no light on any other interesting examples, I have given the result only for supersolvable groups.

\section{REFERENCES}

1. B. Huppert, Monomiale Darstellung endlicher Gruppen, Nagoya Math. J. 6(1953), 93-94. MR 15, 503.

UNIVERSITY OF ILLINOIS, URBANA, ILLINOIS 61801 